\title{
Effects of rosuvastatin on the immune system in healthy volunteers with normal serum cholesterol
}

\author{
Peer W.F. Karmaus, ${ }^{1}$ Min Shi, ${ }^{2}$ Shira Perl, ${ }^{3}$ Angélique Biancotto, ${ }^{3}$ Julián Candia, ${ }^{3}$ Foo Cheung, ${ }^{3}$ \\ Yuri Kotliarov, ${ }^{3}$ Neal Young, ${ }^{4}$ Michael B. Fessler, ${ }^{1}$ and the $\mathrm{CHI}$ Consortium ${ }^{5}$
}

IImmunity, Inflammation, and Disease Laboratory, ${ }^{2 B i o s t a t i s t i c s ~ \& ~ C o m p u t a t i o n a l ~ B i o l o g y ~ B r a n c h, ~ N a t i o n a l ~ I n s t i t u t e ~ o f ~}$ Environmental Health Sciences, Research Triangle Park, North Carolina, USA. ${ }^{3}$ Trans-NIH Center for Human Immunology, Autoimmunity, and Inflammation, NIH, Bethesda, Maryland, USA. ${ }^{4}$ Hematology Branch, National Heart, Lung, and Blood Institute, $\mathrm{NIH}$, Bethesda, Maryland, USA. ${ }^{5}$ The $\mathrm{CHI}$ Consortium is detailed in the supplemental material.

BACKGROUND. HMG-CoA reductase inhibitors (statins) are prescribed to millions of people. Statins are antiinflammatory independent of their cholesterol-reducing effects. To date, most reports on the immune effects of statins have assayed a narrow array of variables and have focused on cell lines, rodent models, or patient cohorts. We sought to define the effect of rosuvastatin on the "immunome" of healthy, normocholesterolemic subjects.

METHODS. We conducted a prospective study of rosuvastatin ( $20 \mathrm{mg} / \mathrm{d} \times 28$ days) in 18 statin-naive adults with $\mathrm{LDL}$ cholesterol $<130 \mathrm{mg} / \mathrm{dL}$. A panel of $>180 \mathrm{immune} /$ biochemical/endocrinologic variables was measured at baseline and on days 14, 28, and 42 (14 days after drug withdrawal). Drug effect was evaluated using linear mixed-effects models. Potential interactions between drug and baseline high-sensitivity C-reactive protein (hsCRP) were evaluated.

RESULTS. A wide array of immune measures changed (nominal $P<0.05$ ) during rosuvastatin treatment, although the changes were modest in magnitude, and few met an FDR of 0.05. Among changes noted were a concordant increase in proinflammatory cytokines (IFN- $\gamma$, IL-1及, IL-5, IL-6, and TNF- $\alpha$ ) and peripheral blood neutrophil frequency, and a decline in activated Treg frequency. Several drug effects were significantly modified by baseline hsCRP, and some did not resolve after drug withdrawal. Among other unexpected rosuvastatin effects were changes in erythrocyte indices, glucose-regulatory hormones, CD8 ${ }^{+}$T cells, and haptoglobin.

CONCLUSION. Rosuvastatin induces modest changes in immunologic and metabolic measures in normocholesterolemic subjects, with several effects dependent on baseline CRP. Future, larger studies are warranted to validate these changes and their physiological significance.

TRIAL REGISTRATION. ClinicalTrials.gov NCT01200836.

FUNDING. This research was supported by the Intramural Research Program of the NIH, National Institute of Environmental Health Sciences (Z01 ES102005), and the trans-NIH Center for Human Immunology.

Conflict of interest: SP is an

employee of AstraZeneca.

Copyright: () 2019, American Society for Clinical Investigation.

Submitted: July 3, 2019 Accepted: September 25, 2019 Published: November 1, 2019

Reference information: /CI Insight. 2019;4(21):e131530. https://doi.org/10.1172/jci. insight.131530.

\section{Introduction}

HMG-CoA reductase inhibitors (statins) are prescribed to more than one-quarter of adults over 40 years of age in the United States for atherosclerotic cardiovascular disease (ASCVD) treatment or prophylaxis (1). In addition to decreasing serum cholesterol levels, statins have antiinflammatory and immunomodulatory properties (2), suggesting their potential for wider disease application. Most studies on the immune effects of statins, however, have been performed either in rodent models, commonly using very high doses delivered parenterally $(3,4)$, or in in vitro cell-based studies, using supraphysiologic concentrations (5-7). Reported effects of statins on the human immune system in vivo have largely derived from studies of patients with hypercholesterolemia, coronary artery disease, and/or HIV infection (8-12) and are thus of 
Table 1. Baseline characteristics of study subjects

\begin{tabular}{|c|c|c|}
\hline & \multicolumn{2}{|c|}{ Study subgroup } \\
\hline & CRP-high $(n=7)$ & CRP-low $(n=11)^{A}$ \\
\hline Age (yr, mean [SD]) & $38.64(10.38)$ & $42.86(10.54)$ \\
\hline \multicolumn{3}{|l|}{ Sex } \\
\hline Female, $n$ & 4 & 6 \\
\hline Male, $n$ & 3 & 5 \\
\hline \multicolumn{3}{|l|}{ Race } \\
\hline Asian, $n$ & 0 & 2 \\
\hline Black, $n$ & 3 & 1 \\
\hline White, $n$ & 4 & 8 \\
\hline hsCRP (mg/L, mean [SD]) & $6.53(5.37)$ & $0.65(0.66)$ \\
\hline Total cholesterol (mg/dL, mean [SD]) & $176.14(21.14)$ & $186.50(32.79)$ \\
\hline LDL-C (mg/dL, mean [SD]) & $98.71(19.73)$ & $106.00(29.19)$ \\
\hline HDL-C (mg/dL, mean [SD]) & $56.86(10.40)$ & $61.90(16.42)$ \\
\hline Triglycerides (mg/dL, mean [SD]) & $102.00(57.50)$ & $93.00(63.35)$ \\
\hline
\end{tabular}

uncertain generalizability. In this context, different statins have been reported to reduce serum proinflammatory cytokines (e.g., TNF- $\alpha$, IL-6, IL-8), C-reactive protein (CRP), and T cell and monocyte activation $(8,11,13)$, some of these effects within $6-8$ weeks $(8,14)$ or earlier $(9)$ and in a manner independent of serum cholesterol reduction (15). There is a great deal of heterogeneity across these clinical reports, however, including some that failed to observe any immune effects $(10,12,16)$. Moreover, some cell-based reports have even indicated the potential for statins to induce proinflammatory cytokine production by human leukocytes $(17,18)$, prompting recent calls to determine whether this occurs in vivo (19). Given the potential public health implications, there is a critical need to better resolve the effects of this widely used class of drugs on the human immune system.

To date, few reports have examined the in vivo immune effects of statins in healthy, normocholesterolemic subjects, and these studies have measured a narrow array of immune markers. One group found that simvastatin and atorvastatin had opposite effects on $\mathrm{T}$ cell activation within 14 days of treatment but no effect on serum cytokines or high-sensitivity CRP (hsCRP) (20), whereas another group found that simvastatin reduced serum monocyte chemoattractant protein-1 within this time frame (21). Atorvastatin and lovastatin were reported to increase Tregs within 30 days in healthy subjects (22). Notably, the JUPITER trial showed that rosuvastatin reduced major ASCVD events and serum hsCRP in more than 17,000 subjects with baseline LDL cholesterol (LDL-C) $<130 \mathrm{mg} / \mathrm{dL}$ and hsCRP $\geq 2 \mathrm{mg} / \mathrm{L}$ but did not report any additional immune measures (23). Smaller studies in subjects with hypercholesterolemia and HIV have found that suppressive effects of statins on hsCRP (24) and T cell activation (11) were restricted to those with baseline elevations in hsCRP and $\mathrm{T}$ cell activation, respectively. Whether baseline inflammatory status determines effects of statins in healthy subjects is unknown.

Here, we report the results of a prospective 4-week study of rosuvastatin, $20 \mathrm{mg}$ daily, in 18 participants with baseline LDL-C $<130 \mathrm{mg} / \mathrm{dL}$, followed by 2 weeks of drug washout. A comprehensive panel of >180 immune, hematologic, and biochemical measures was evaluated at baseline, during treatment, and after drug discontinuation. Rosuvastatin induced wide-ranging effects. Of interest, we identified several proinflammatory cytokines that appeared to increase during statin treatment and then decreased after statin discontinuation. A subset of drug effects differed significantly between subjects with baseline hsCRP $\geq 2 \mathrm{mg} / \mathrm{L}$ versus $<2 \mathrm{mg} / \mathrm{L}$, suggesting interaction with inflammatory status. Taken together, this is the first report to our knowledge of the in vivo impact of a statin on the immunome of normocholesterolemic, healthy subjects, identifying several potential drug effects that warrant further investigation. 


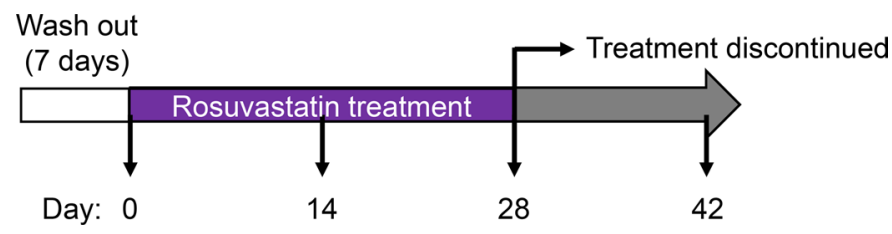

Figure 1. Study design for evaluating the effect of rosuvastatin initiation and discontinuation on the human immunome.

\section{Results}

In order to define the effect of statins on the immunome of healthy, normocholesterolemic subjects, we recruited 18 statin-naive (within 6 months prior to enrollment) adults with LDL-C $<130 \mathrm{mg} / \mathrm{dL}$. Although the original objective was to recruit 20 participants, 10 with normal $(<2 \mathrm{mg} / \mathrm{L})$ and 10 with elevated $(\geq 2$ $\mathrm{mg} / \mathrm{L}$ ) hsCRP, only 7 high-hsCRP subjects could be identified despite extension of the screening period. Baseline characteristics of the study participants are shown in Table 1. Following an initial 7-day washout period during which participants were asked to refrain from nonsteroidal antiinflammatory drugs, antihistamines, steroids (including topical), and niacin formulations, a baseline panel of immune, hematological, and endocrine measurements was collected (Supplemental Table 1; supplemental material available online with this article; https://doi.org/10.1172/jci.insight.131530DS1) (25). Subjects were then commenced immediately on a 28-day course of $20 \mathrm{mg}$ rosuvastatin daily. As shown in Figure 1, the panel of measurements was repeated on days 14, 28, and 42 (i.e., 14 days after drug washout).

Baseline immune profile of subjects with normal versus elevated hsCRP. CRP has been proposed as a clinically robust biomarker for identifying subjects with increased systemic inflammation, and an hsCRP cut point of $2 \mathrm{mg} / \mathrm{L}$ has been proposed (26). Fifty-one measures were found to differ significantly $(P<0.05)$ at baseline between subjects with normal and elevated hsCRP (47 of 51 of these represented increases in subjects with CRP $\geq 2 \mathrm{mg} / \mathrm{L}$ ) (Supplemental Table 2). Of these, 19 met an FDR threshold of 0.05. Consistent with previous reports of the utility of $\mathrm{CRP}$ as a proinflammatory biomarker, several proinflammatory cytokines (IFN- $\gamma$, TNF- $\alpha$, IL-6, IL-12p70, IL-17, IL-18) and chemokines (IL-8, GRO $\alpha$, MIP-1 $\alpha$, MIP-1 $\beta$, RANTES) were elevated in high-hsCRP subjects. Type 2 (IL-4, IL-5, IL-9, IL-13) inflammatory mediators and the antiinflammatory cytokine IL-10 were also elevated in high-hsCRP subjects, suggesting a complex, mixed inflammatory milieu with compensatory signals. Consistent with their higher serum granulocyte-CSF and neutrophilic chemokines, leukocyte differentials of high-hsCRP subjects tended to show increased neutrophils and decreased lymphocytes. High-hsCRP subjects also had higher insulin, C-peptide, glucagon, and leptin than normal hsCRP counterparts, consistent with metabolic stress. Random forest analysis identified IL- 6 and IL-4 as the measures of highest importance for discriminating between CRP-high and CRP-low subjects (Supplemental Figure 1). Taken together, the results indicate that an hsCRP cut point of $2 \mathrm{mg} / \mathrm{L}$ distinguished subjects displaying an associated cluster of low versus high proinflammatory measures.

Measures altered during rosuvastatin treatment. As expected, and confirming a rosuvastatin effect, total cholesterol and LDL-C were significantly reduced in all subjects while on rosuvastatin, with rebound to baseline after drug discontinuation (Figure 2 and Table 2). Triglycerides were also reduced, whereas HDL cholesterol (HDL-C) was unchanged. The effect on serum lipids was seen on day 14 and occurred equivalently in low- and high-hsCRP subjects.

Although several measures from the immunophenotypic panel met nominal statistical significance $(P$ $<0.05$ ), the only other measure to meet an FDR $<0.05$ threshold was the reduction in mean corpuscular hemoglobin concentration (MCHC) (i.e., the concentration of hemoglobin within erythrocytes) (Table 2). Concordant with this, rosuvastatin treatment was also associated with a reduction in hemoglobin mass and mean corpuscular hemoglobin mass, and with an increase in mean corpuscular volume (MCV), collectively suggesting independent effects on hemoglobin and erythrocyte size. Erythrocyte count was also depressed by rosuvastatin, whereas hematocrit, while reduced, was not significant degree $(P=0.09)$. Arguing against induction of hemolysis (27), haptoglobin was significantly increased during rosuvastatin administration, followed by normalization after rosuvastatin discontinuation (Table 2 and Supplemental Figure 2A).

Of interest, several proinflammatory cytokines (IFN- $\gamma$, IL-1 $\beta$, IL-5, IL-6, IL-15) increased, albeit modestly, in subjects while on rosuvastatin (nominal $P<0.05$ ) (Table 2 and Figure $3, \mathrm{~A}-\mathrm{E}$ ). Both IL-18, like IL-1 $\beta$, a product of the inflammasome $(17,18)$, and TNF, an inflammasome-independent 

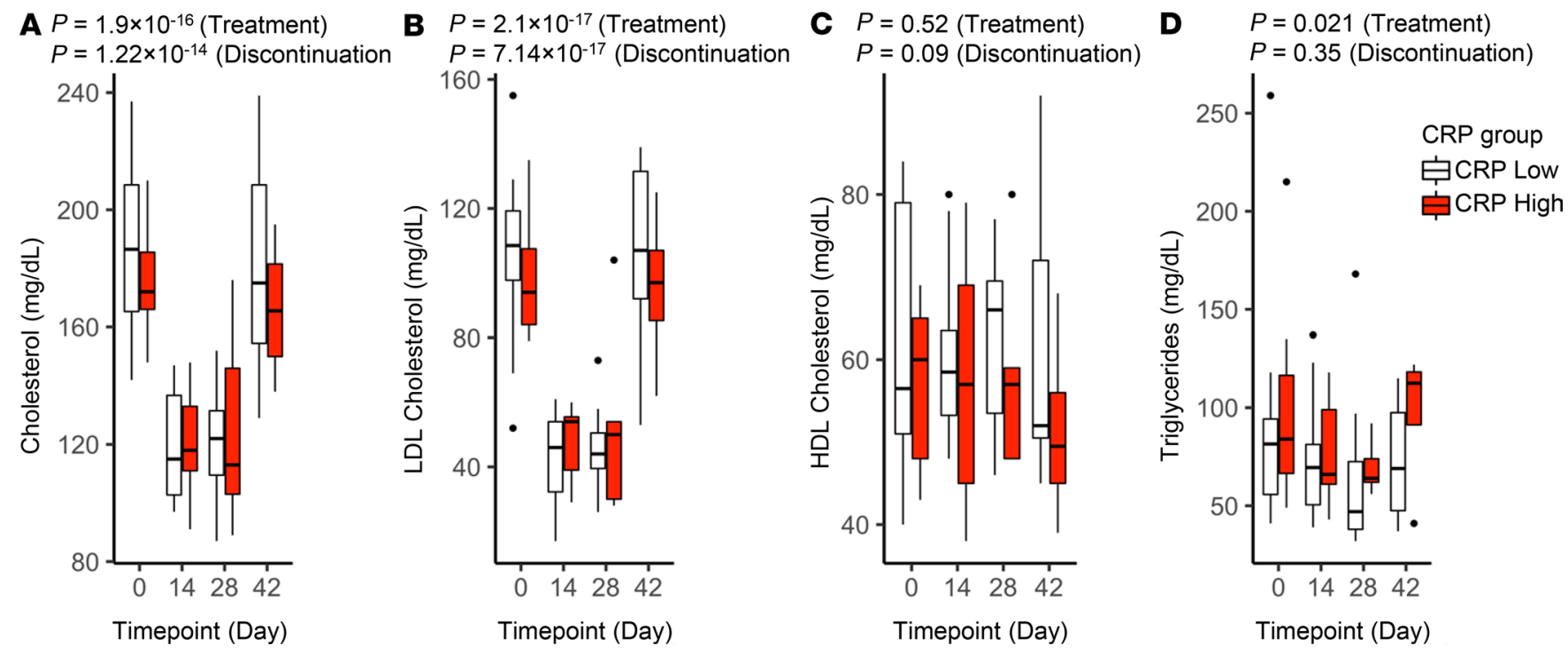

Figure 2. Effect of rosuvastatin on serum lipids. Serum total cholesterol (A), LDL-C (B), HDL-C (C), and triglycerides (D) were measured in study participants at the indicated trial time points (baseline [day 0], rosuvastatin treatment [days 14 and 28], and 14 days after rosuvastatin discontinuation [day 42]). Data for subjects with low versus high CRP at baseline are plotted separately. Boxes depict IQR around the median. The upper whisker extends from the hinge to the largest value no further than $1.5 *$ IQR from the hinge; the lower whisker extends from the hinge to the smallest value at most $1.5 *$ IQR of the hinge. Outlying points are plotted individually. Nominal $P$ values for rosuvastatin treatment and discontinuation were determined for the overall study group by linear regression (also listed in Table 2 and Table 3).

cytokine, also increased in response to rosuvastatin treatment, but these differences did not achieve statistical significance (Supplemental Figure 2, B and C). Formal correlation analysis revealed that all of the aforementioned cytokines, along with several others (IL-2, IL-4, IL-7, IL-8, IL-12p70), were strongly correlated in their response over time to rosuvastatin (Supplemental Figure 3). By contrast, serum total cholesterol and LDL-C showed no correlation with this cytokine module. Although in most cases the increase in cytokine levels was more marked in CRP-high subjects, formal testing did not reveal a significant interaction between rosuvastatin and baseline hsCRP (Supplemental Table 3). In parallel with the increases in these proinflammatory mediators, rosuvastatin induced an increase in the antiinflammatory cytokine IL-10 in CRP-high subjects $(P=0.05)$ (Supplemental Figure 2D) and in the antiprotease and anticoagulant protein $\alpha 2$-macroglobulin (A2M) (Table 2). Although modeling indicated that rosuvastatin was associated with a reducing effect on CRP, this did not achieve statistical significance $(P=0.08)$.

With regard to leukocyte subpopulations, rosuvastatin treatment was associated with an increase in neutrophil frequency in both CRP groups (Table 2 and Figure 3F). By contrast, we observed a decline in the frequency of activated Tregs $\left(\mathrm{CD} 4{ }^{+} \mathrm{CD} 127^{\mathrm{lo}} \mathrm{CD} 25^{\text {hi }} \mathrm{CCR} 4^{+} \mathrm{HLA}-\mathrm{DR}{ }^{+}\right.$cells) that was transient in CRPhigh subjects but sustained in CRP-low subjects (Figure 3G). In addition, a decline was seen in $\mathrm{CD} 127^{10} \mathrm{C}$ $\mathrm{D} 27^{\mathrm{hi}} \mathrm{CD} 39^{+} \mathrm{HLA}-\mathrm{DR}^{+}$Tregs during rosuvastatin administration. Rosuvastatin was also associated with a decline in some naive $\mathrm{CD}^{+} \mathrm{T}$ cell populations, such as $\mathrm{CD} 28^{-} \mathrm{CCR} 7^{+} \mathrm{CD} 45 \mathrm{RA}{ }^{+}$cells.

Complex effects were observed among $\mathrm{CD}^{+} \mathrm{T}$ cell subpopulations and $\mathrm{B}$ cells. Rosuvastatin treatment was associated with a reduction in $\mathrm{CD} 28^{+} \mathrm{CCR} 7^{-} \mathrm{CD} 45 \mathrm{RA}^{+} \mathrm{CD} 8^{+} \mathrm{T}$ cells irrespective of CRP status (Table 2), whereas reductions in HLA-DR ${ }^{+} \mathrm{CD} 8^{+} \mathrm{T}$ cells and in $\mathrm{CD} 38^{+} \mathrm{CD} 8^{+} \mathrm{T}$ cells were seen only in CRP-high subjects (Figure 3, H and I), as confirmed by formal interaction testing (Supplemental Table 3). This CRP-selective effect on $\mathrm{CD}^{+} \mathrm{T}$ cells interestingly occurred despite the $2 \mathrm{CD} 8^{+} \mathrm{T}$ cell subsets displaying reciprocal relationships to $\mathrm{CRP}\left(\mathrm{CD} 38^{+}\right.$cells elevated in high-CRP subjects, HLA-DR ${ }^{+}$cells reduced in CRP-high subjects; Table 2 and Figure 3). By contrast, the CD28 $8^{+} \mathrm{CCR} 7^{+} \mathrm{CD} 45 \mathrm{RA}^{-}$subset of CD8 ${ }^{+} \mathrm{T}$ cells was increased on rosuvastatin irrespective of CRP status $(P=0.05)$. Finally, CD19+CD20-CD38CD24- B cells were noted to increase significantly in response to rosuvastatin treatment only in CRP-high subjects (Figure 3J), as confirmed by formal interaction testing (Supplemental Table 3). Taken together, the results indicate that rosuvastatin administration was associated with complex changes in circulating myeloid and lymphoid populations. 
Table 2. Variables changed by rosuvastatin treatment in overall study group

\begin{tabular}{|c|c|c|c|c|c|c|c|}
\hline & $\begin{array}{c}\text { Day 0, } \\
\text { median, } \\
\text { all (CRP'o, CRPhi) }\end{array}$ & $\begin{array}{c}\text { Day 14, } \\
\text { median, } \\
\text { all (CRP'o, CRPhi) }\end{array}$ & $\begin{array}{c}\text { Day 28, } \\
\text { median, } \\
\text { all (CRP'o, CRPh) }\end{array}$ & Estimate & SE & $\operatorname{Pr}(\geq|t|)^{A}$ & FDR \\
\hline LDL-C & $106(109,94)$ & $48(46,54)$ & $45(44,50)$ & -0.82 & 0.06 & $2.1 \times 10^{-17}$ & $3.99 \times 10^{-15}$ \\
\hline $\mathrm{MCHC}$ & $34.3(34.3,33.9)$ & $33.7(33.7,34.2)$ & $33.9(33.9,33.8)$ & -0.40 & 0.10 & $2.8 \times 10^{-4}$ & 0.018 \\
\hline Hemoglobin & $13.9(13.4,14)$ & $13.5(13.35,13.5)$ & $13.4(13.3,13.7)$ & -0.39 & 0.13 & $3.2 \times 10^{-3}$ & 0.155 \\
\hline MCV & $88.25(88.6,87.2)$ & $89.6(89.7,88.4)$ & $89.1(89.4,88.7)$ & 0.54 & 0.20 & $8.9 \times 10^{-3}$ & 0.247 \\
\hline RBC & $4.68(4.48,4.70)$ & $4.49(4.37,4.60)$ & $4.42(4.42,4.67)$ & -0.11 & 0.04 & 0.013 & 0.327 \\
\hline IL-1 $\beta$ & $3.08(2.63,4.70)$ & $3.16(2.94,4.54)$ & $3.23(2.73,13.44)$ & 0.17 & 0.07 & 0.019 & 0.346 \\
\hline Triglycerides & $84.0(81.5,84.0)$ & $66.0(69.5,66.0)$ & $59.0(47.0,64.0)$ & -0.21 & 0.09 & 0.021 & 0.346 \\
\hline $\mathrm{X} 116^{\mathrm{C}}$ & $1.43(1.56,1.22)$ & $1.21(1.31,1.21)$ & $1.28(1.40,1.06)$ & -0.14 & 0.06 & 0.026 & 0.346 \\
\hline$A 2 M\left(\times 10^{5}\right)$ & $1.75(1.76,16.2)$ & $1.98(2.10,1.97)$ & $1.86(1.87,1.85)$ & 0.15 & 0.07 & 0.035 & 0.346 \\
\hline MPV & $11.0(11.1,10.1)$ & $10.8(10.8,10.1)$ & $10.8(11.0,10.2)$ & -0.18 & 0.08 & 0.036 & 0.346 \\
\hline SCGF $-\beta\left(\times 10^{3}\right)$ & $5.62(3.58,9.67)$ & $5.91(5.00,14.58)$ & $6.12(5.40,17.00)$ & 0.25 & 0.12 & 0.037 & 0.346 \\
\hline NK cells & $269(182,280)$ & $218(211,221)$ & $212(182,238)$ & -26.65 & 12.37 & 0.037 & 0.346 \\
\hline IFN- $\gamma$ & $\begin{array}{c}217.11 \\
(169.25,259.99)\end{array}$ & $\begin{array}{c}215.04 \\
(164.08,340.11)\end{array}$ & $\begin{array}{c}199.93 \\
(177.50,2608.55)\end{array}$ & 0.15 & 0.07 & 0.038 & 0.346 \\
\hline $\mathrm{X} 106^{\mathrm{D}}$ & $11.4(10.0,13.1)$ & $7.47(7.20,14.3)$ & $10.8(7.83,12.6)$ & -0.14 & 0.07 & 0.040 & 0.346 \\
\hline Neutrophils (\%) & $54.3(51.9,62.1)$ & $57.0(50.9,67.0)$ & $59.4(58.1,64.7)$ & 3.60 & 1.75 & 0.045 & 0.346 \\
\hline Insulin & $\begin{array}{c}317.36(288.03 \\
476.72)\end{array}$ & $\begin{array}{c}314.23(278.88 \\
524.56)\end{array}$ & $336.87(320.03,625.38)$ & 0.23 & 0.11 & 0.046 & 0.346 \\
\hline
\end{tabular}

AVariables that were significantly changed in overall study group (nominal $P$ value $[\operatorname{Pr}(\geq|t|)] \leq 0.05$ ) after commencement of rosuvastatin, as assessed by linear regression. FDR-adjusted $P$ values are also shown. Median values for analytes on specific study days are shown for all subjects, for CRP-low subjects, and for CRP-high subjects and may be adjusted as indicated (e.g., $\left.\times 10^{\times}\right)$. Where not otherwise indicated, median values are in relative units. ${ }^{{ }^{-} C D 3^{+} C D 4^{+} C D 8}$

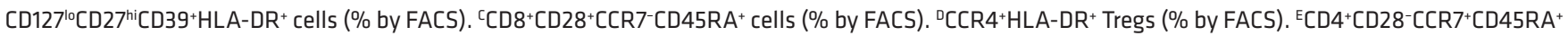
Tregs (\% by FACS). IL-1RA, IL-1 receptor antagonist; MCH, mean corpuscular hemoglobin; MPV, mean platelet volume; SE, standard error.

Among metabolic measures, glucagon and glucagon-like peptide 1 (GLP-1) exhibited a similar pattern of response to rosuvastatin, modestly increasing by day 28 and then declining after drug discontinuation (Table 2, Table 3, and Figure 4, A and B). Of interest, these 2 measures were highly correlated in their response pattern to the cytokine module noted above (Supplemental Figure 3). Insulin and C-peptide both progressively increased on rosuvastatin $(P=0.05)$, especially in CRP-high subjects, and their levels did not normalize after drug discontinuation (Figure 4, C and D).

Measures altered after rosuvastatin withdrawal. By linear mixed-effects modeling, 31 measures were nominally statistically significant $(P<0.05)$ for an effect of rosuvastatin discontinuation; of these, 5 met an FDR $<0.05$ threshold (Table 3). Several measures, including glucagon, GLP-1, and haptoglobin exhibited a reciprocal response to rosuvastatin initiation (increase) versus discontinuation (decrease), suggesting a temporal and reversible effect of the drug. Of the proinflammatory measures that increased while on rosuvastatin, several were noted to decline after statin discontinuation (IFN- $\gamma$, TNF- $\alpha$, IL- $1 \beta$, IL-5, IL-6, IL-15) (Figure 3), also temporally consistent with a causal effect of the drug; however, of these, only the decline in IL-1 $\beta$ achieved statistical significance, and this effect was restricted to the high- 


\section{Rosuvastatin Treatment Effect}

A

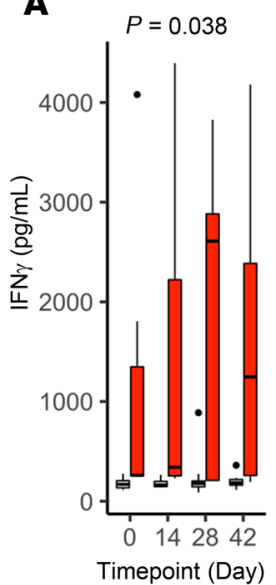

B

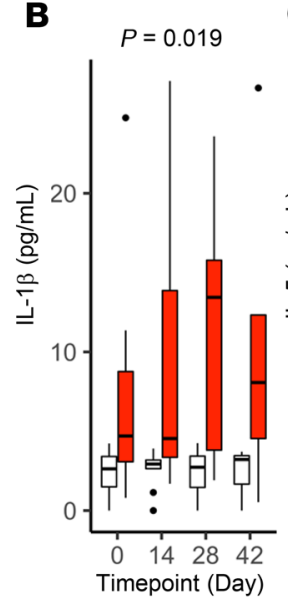

c
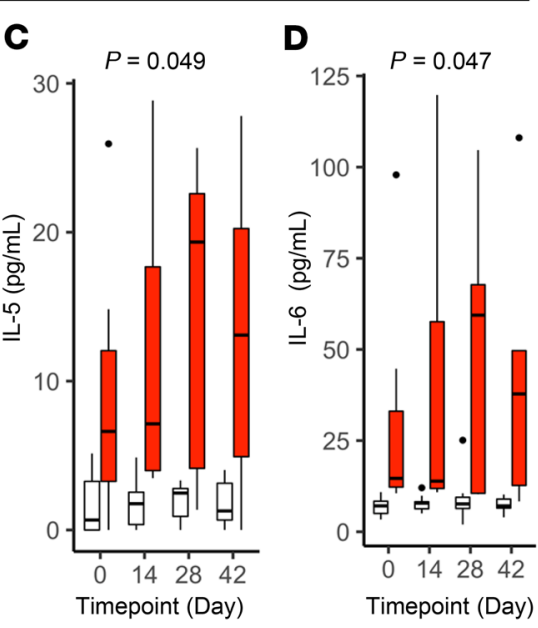

Figure 3. Effect of rosuvastatin on immune measures. Immune measures were plotted in study participants at the indicated trial time points (baseline [day 0], rosuvastatin treatment [days 14 and 28], and 14 days after rosuvastatin discontinuation [day 42]). Data for subjects with low versus high CRP at baseline are plotted separately. Boxes depict IQR around the median. Upper whisker extends from the hinge to the largest value no further than $1.5^{*}$ IQR from the hinge; lower whisker extends from the hinge to smallest value at most $1.5^{*}$ IQR of the hinge. Outlying points are plotted individually. Nominal $P$ values for the overall study group were determined by linear regression. $P$ values in $\mathbf{A}-\mathbf{G}$ are for rosuvastatin treatment compared with day 0 (Table 2); in $\mathbf{H}-\mathbf{J}$, for rosuvastatin treatment in interaction with CRP (Supplemental Table 3); and in $\mathbf{K}$ and $\mathbf{L}$, for rosuvastatin discontinuation (Table 3 ).
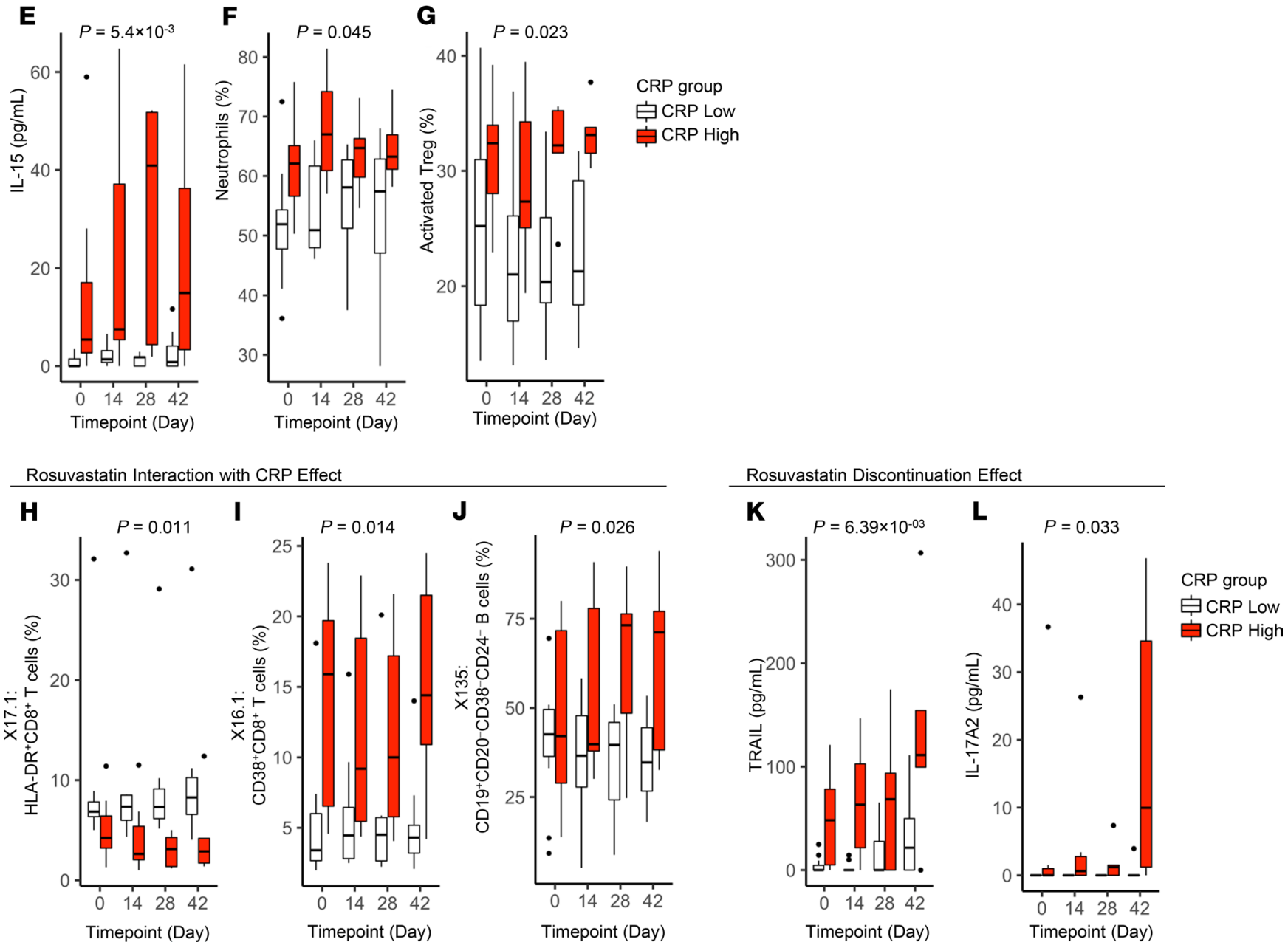

CRP group (Supplemental Table 4 and Figure 3B). A total of 17 measures exhibited a statistically significant interaction of the drug discontinuation effect with baseline hsCRP status (Supplemental Table 4).

Additional patterns of drug discontinuation effect were noted. Some immune measures were noted to increase significantly after statin discontinuation despite not having changed significantly from baseline during statin therapy. Among these were TRAIL and IL-17A2 (Table 3 and Figure 3, K and L), the latter of which was noted to interact with CRP (i.e., only to increase in CRP-high subjects; Supplemental Table 4 and Figure 3L). Of interest, IL-25 (i.e., IL-17E) was also noted to increase after 
Table 3. Variables changed by rosuvastatin discontinuation in the overall study group

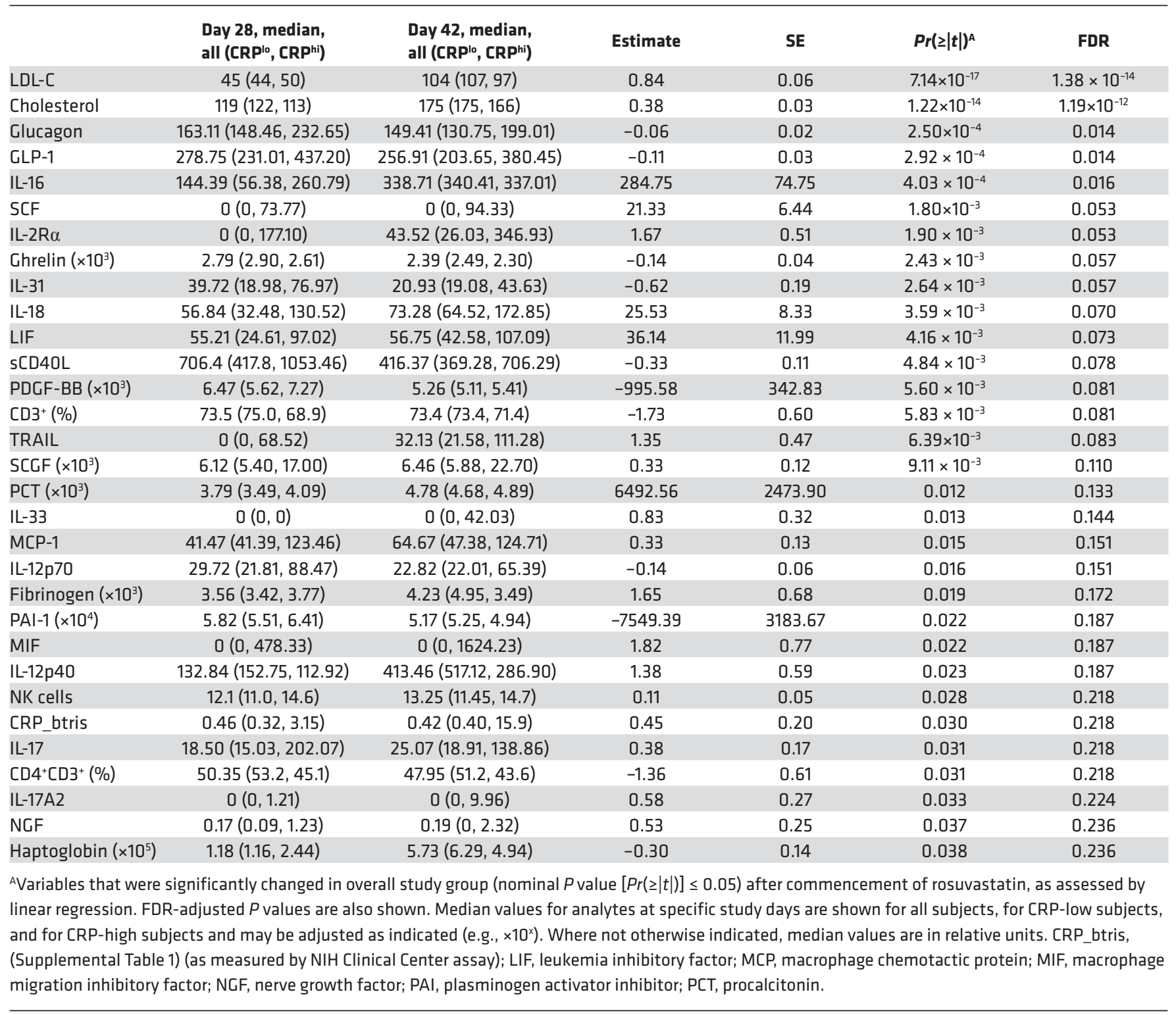

statin discontinuation in interaction with CRP (Supplemental Table 4). Other measures increased from baseline during statin administration and then further increased after statin administration (IL-18, stem cell growth factor $\beta$ [SCGF- $\beta$ ]) (Table 3 and Supplemental Figure 2, B and E).

\section{Discussion}

Statins modulate the function of multiple immune cell types (2). Many of these effects have been shown to arise from reduced prenylation of signaling proteins (due to statin-mediated depletion of cellular isoprenoids) or reduced cell cholesterol (2-4), but antiinflammatory effects that are independent of HMG CoA reductase have also been identified (e.g., inhibition of LFA-1 and HDAC-2; refs. 28, 29). Additional antiinflammatory or proresolving actions that have been identified for statins include induction of 15-epi-lipoxin A4 (30) and PPAR $\gamma$ (21). The majority of the reports on immune effects of statins were derived from studies using cell lines, rodents, or human cells studied ex vivo. Studies of statin-treated humans have largely arisen from patient cohorts with hypercholesterolemia, HIV infection, or ASCVD and have generally focused on 

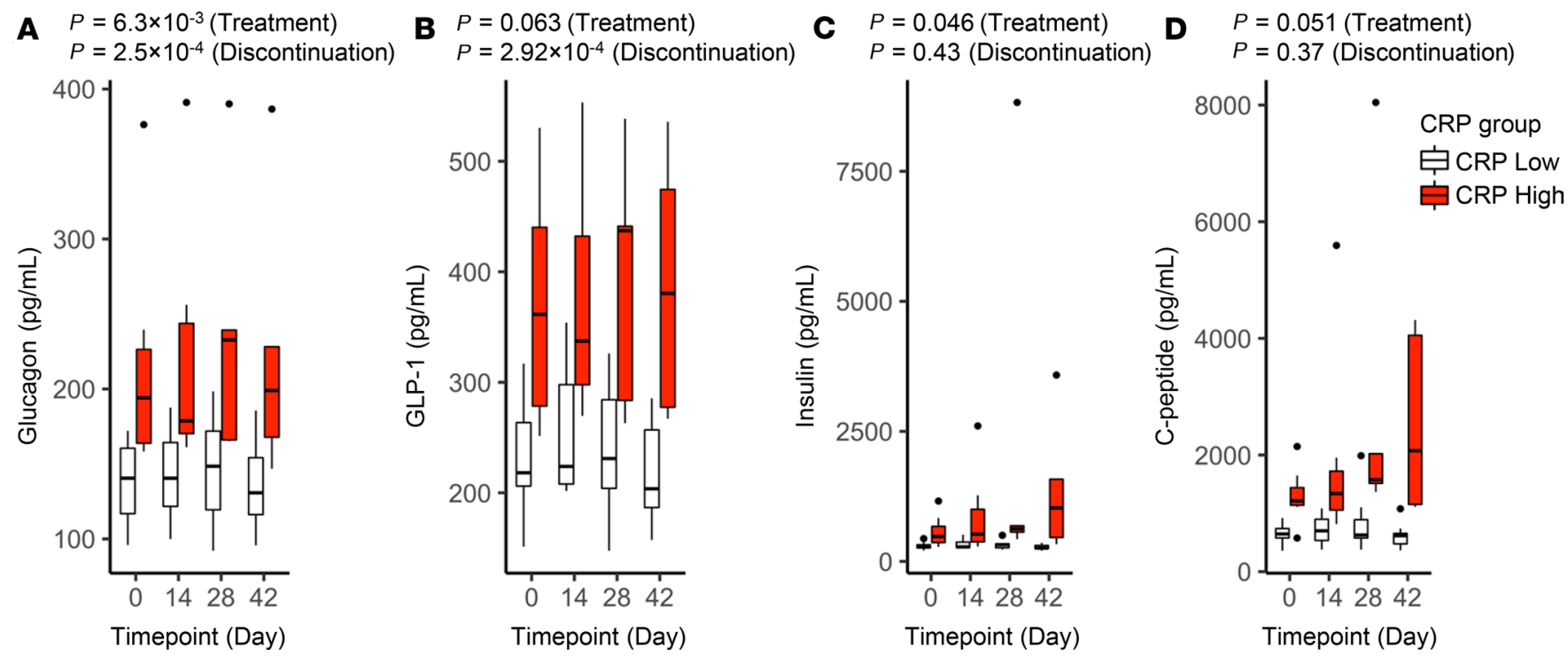

Figure 4. Effect of rosuvastatin on endocrine measures. Levels of glucagon (A), GLP-1 (B), insulin (C), and C-peptide (D) in study subjects during the rosuvastatin time course. Subjects with low versus high CRP at baseline are plotted separately. The upper whisker extends from the hinge to the largest value no further than 1.5 * IQR from the hinge; the lower whisker extends from the hinge to the smallest value at most $1.5 *$ IQR of the hinge. Outlying points are plotted individually. Nominal $P$ values for rosuvastatin treatment and discontinuation were determined for the overall study group by linear regression (also listed in Table 2 and Table 3).

select immune readouts (8-12). Defining the immune effects of statins in broader populations is important, as statins are increasingly being considered in normocholesterolemic subjects at risk for ASCVD (23) as well as in a growing list of immunologic human disorders, including asthma, acute respiratory distress syndrome (ARDS), pneumonia, sepsis, chronic obstructive pulmonary disease, rheumatoid arthritis, lupus, multiple sclerosis, graft-versus-host disease, and organ transplantation rejection (31). Moreover, there is increasing interest in the effects of statins on infection risk and vaccination efficacy at a population level (2).

Here, we report what we believe to be the first prospective comprehensive immunologic and biochemical study profiling statin-treated normocholesterolemic individuals. We have cataloged a large number of putative statin effects, many of which are, to our knowledge, previously unreported. Given that few of these changes met a FDR threshold of 0.05 , the results should be considered hypothesis generating in nature. Contrary to some (24), but not all (20), prior reports, we did not detect a decrease in hsCRP with rosuvastatin. Rosuvastatin discontinuation was, however, associated with an increase in hsCRP (Table 3).

Of interest, we found that rosuvastatin treatment was associated with concordant, albeit modest, upregulation of several proinflammatory indicators, including cytokines (IFN- $\gamma$, IL-1 $\beta$, IL-5, IL-6), and peripheral blood neutrophil frequency. Most reports of ex vivo treated human leukocytes and of ex vivo assays on in vivo treated leukocytes have found that statins reduce proinflammatory cytokines (32-34). Simvastatin was also found to reduce serum cytokines within 2 weeks in normocholesterolemic subjects (21). By contrast, ex vivo treatment of human monocytes with lipophilic statins (simvastatin, atorvastatin, lovastatin) but not pravastatin induced upregulation of multiple cytokines that was blocked by cotreatment with mevalonic acid (35). Simvastatin has also been reported to induce IL-18, TNF- $\alpha$, and IFN in human peripheral blood mononuclear cells (36), to augment LPS induction of multiple cytokines in human peripheral blood mononuclear cells (18), and to promote caspase-1-dependent processing of IL-1 $\beta$ in THP-1 cells through a mechanism involving prenylation inhibition (37). Similarly, fluvastatin reportedly induces caspase-1-dependent release of mature IL-1 $\beta$ through a mechanism involving ATP release (38), and pravastatin induces IL-1 $\beta$ and IL-18 in macrophages through induction of mitochondrial reactive oxygen species (39). A recent report also found that ARDS patients treated with rosuvastatin tended to have higher plasma levels of IL-18 (40). Taken together with these results, our finding that rosuvastatin treatment is associated with increases in both caspase-1-dependent (inflammasome) (IL-1 $\beta$, IL-18) and -independent (IFN- $\gamma$, TNF- $\alpha$, IL-5, IL-6, IL-15) cytokines is thus consistent with prior reports on rosuvastatin and other statins. The reason for divergent findings for statins in inflammation is unclear but may possibly relate to 
technical/biological differences across reports, including the specific statin formulation studied, as well as the basal inflammatory state of the cells/subjects studied. Our finding that the upregulation of several mediators by rosuvastatin was more pronounced in subjects with high-hsCRP status is consistent with the latter possibility. A recent reanalysis of the HARP-2 trial found that only those ARDS subjects with a "hyperinflammatory" subphenotype benefited clinically from treatment with simvastatin (41). Somewhat similar to this, we found that several immune effects of rosuvastatin were dependent on baseline hsCRP (Supplemental Tables 3 and 4), with several of these effects more pronounced in CRP-high subjects.

Our findings of reduced hemoglobin, MCHC, and erythrocyte number, and increased erythrocyte volume $(\mathrm{MCV})$ during rosuvastatin treatment suggest complex effects of rosuvastatin on RBCs. In an analysis of US national survey data, we previously reported that non-HDL-C is directly related to serum hemoglobin and erythrocyte number, whereas HDL-C is directly related to $\mathrm{MCV}$, suggesting communication between cholesterol and erythrocyte biology (42). Rosuvastatin has been reported to reduce the cholesterol content of erythrocyte membranes and to increase their fluidity/deformability (43-46). Moreover, statins alter erythrocyte $\mathrm{Na}^{+} / \mathrm{K}^{+}$-ATPase activity and ATP release $(45,46)$. A post hoc analysis of the JUPITER trial did not find any effect of rosuvastatin on hemoglobin mass (47), nor did a study of a 24-week course of atorvastatin in 81 hypercholesterolemic subjects (48); however, neither study reported MCHC or MCV.

Some prior reports have suggested that statins may increase glucose intolerance or even increase the incidence of diabetes mellitus $(49,50)$. The magnitude of this risk and its underlying mechanism are both poorly defined. Of interest, glucagon significantly increased during rosuvastatin therapy. In parallel, and possibly in compensation, insulin, C-peptide, and the insulinotropic hormone GLP-1 (51) all also increased during rosuvastatin administration. Of the 4, only glucagon and GLP-1 normalized after drug discontinuation. Future studies are warranted to investigate the possibility that statins impact glucose tolerance through alteration of glucagon and/or GLP-1 levels.

Limitations of our report should be noted. Our study is limited by its small size, its 28-day treatment duration, and its focus on a single statin, and thus will require independent validation. The changes we report with rosuvastatin treatment and withdrawal are also modest in magnitude and of uncertain physiologic significance, and most did not persist after multiple-testing correction. We also cannot discount the possibility that some of the effects we report could be secondary to drug toxicity (e.g., muscle or hepatic inflammation). In addition, particularly in a small study such as this, interindividual environmental differences such as physical or psychological stress could have influenced the results. It is also possible that the 1 -week washout period was insufficient. Although renal impairment and diabetes mellitus were exclusion criteria, these conditions are not routinely avoided in selection of patients for statin therapy in the clinical arena. Finally, the soluble mediators analyzed by Luminex were run in 2 batches, of which the second comprised 12 measurements made on $3 \mathrm{CRP}$-high subjects. In order to address the possibility of batch effects, we ran a sensitivity analysis adjusting for batch number, but found that this did not materially change the findings for rosuvastatin effect, rosuvastatin discontinuation, or rosuvastatin $\times$ CRP interactions. Specifically, there was no change in the measures found to have $P<0.05$ in Table 3, Supplemental Table 3, or Supplemental Table 4, whereas 2 measures in Table 2 (effect of rosuvastatin treatment) rose marginally above the significance threshold (IL-5 $[P=0.054]$ and IL-6 $[P=0.053]$ ). In a separate analysis, we found that omission of data for the $3 \mathrm{CRP}$-high subjects in batch 2 did substantially reduce the number of significant baseline differences detected between CRP-low and -high subjects (Supplemental Table 5).

Remarkably, subjects receiving the same dose of rosuvastatin or atorvastatin may have as much as a 45 -fold variability in plasma concentration of the drug (52). This variation is almost certainly genetic, as more than 40 genes have been identified to date that affect statin efficacy and safety (53). Although we did not measure serum rosuvastatin levels in study participants, the prompt and sustained reduction in LDL-C observed in all subjects (Figure 2B) strongly suggests that all had therapeutic rosuvastatin levels. As different statins with varying physicochemical properties (e.g, hydrophobicity vs. hydrophilicity) may potentially have distinct biological properties, future studies may be warranted to compare the immune effects of different statin formulations. The complexity of statin pharmacogenomics suggests that the effect of statins on the immune system will be found to be genetically regulated, and perhaps that the differences in statin immune response phenotypes may be more granular than can be achieved by dichotomizing patients by hsCRP level. Although our study was not designed to identify novel inflammatory biomarkers that aid in prediction of statin immune effects, we propose that some of the biomarkers that we found to segregate best between CRP-low and -high subjects - such as IL-6, IL-1R $\alpha$, IFN- $\gamma$, and resistin (Supplemental Table 
2 and Supplemental Figure 1) - may be interesting candidates. Detection of robust effects of statins on the human immunome may also require immune challenge (e.g., vaccination, infection) as suggested by the greater response magnitude of rosuvastatin in CRP-high compared with CRP-low groups (Figure 3 and Supplemental Table 3). Indeed, given that several of the changes seen with rosuvastatin were more prominent in, or restricted to, CRP-high subjects and most were modest in magnitude, one conclusion that could reasonably be drawn from our findings is that rosuvastatin induces minimal changes in the steady-state immunome in normocholesterolemic CRP-low subjects.

In summary, we have performed a comprehensive interrogation of the human immunome in healthy, normocholesterolemic subjects treated for 28 days with rosuvastatin. We report that rosuvastatin in induces a variety of immunologic, biochemical, and endocrinologic changes. Several new lipid-lowering agents that work through distinct mechanisms have either been approved or are currently under regulatory review for use in human subjects (54). Some of these drugs, such as bempedoic acid, have been shown in preliminary studies to reduce hsCRP (54), whereas others, such as anti-PCSK9 antibodies, appear not to do so (55). Given that some of the immunomodulatory and cardioprotective effects of statins in hypercholesterolemic subjects have been shown to be independent of cholesterol lowering (15), it will now be critical to compare the immune effects of statins with these newly emerging drug classes.

\section{Methods}

Clinical protocol. The study was designed to recruit up to 30 healthy individuals with normal serum cholesterol (LDL-C $<130 \mathrm{mg} / \mathrm{dL}$ ), with the intent of enrolling 20 evaluable healthy subjects, 10 of them with hsCRP $\geq 2 \mathrm{mg} / \mathrm{L}$ and 10 with $\mathrm{hsCRP}<2 \mathrm{mg} / \mathrm{L}$, as per the JUPITER trial (23). Inclusion criteria were healthy status (confirmed by history, physical examination, and routine blood work), age $\geq 18$ years, and LDL-C $<130 \mathrm{mg} / \mathrm{dL}$. Exclusion criteria were pregnancy or active lactation, abnormal liver function tests (AST >34 U/L; ALT > $41 \mathrm{U} / \mathrm{L}$; total bilirubin >1.0 mg/dL; alkaline phosphatase >116 U/L), elevated serum creatine kinase, other contraindications to statins (e.g., renal impairment, diabetes mellitus, statin hypersensitivity), and statin usage within 6 months prior to enrollment. Participants were advised not to initiate any new medications, including over-the-counter drugs, during study participation. After study enrollment, subjects underwent a 1-week washout period, during which they were requested to refrain from nonsteroidal antiinflammatory drugs, antihistamines, corticosteroids (including topical agents), and niacin preparations. Medication and supplement use reported by participants at enrollment is shown in Supplemental Table 6. Blood was collected at the end of the 1 -week washout immediately prior to the first dose of a 4-week course of rosuvastatin ( $20 \mathrm{mg}$ daily) and again 2 weeks ( \pm 2 days), 4 weeks ( \pm 2 days; time of rosuvastatin discontinuation), and 6 weeks ( \pm 2 days) later. Participants were questioned about rosuvastatin adherence and pill counts were performed during study visits. Study flow is depicted in Figure 1.

Flow cytometry for comprehensive immunophenotyping. Methods are as previously reported (25). In brief, peripheral blood mononuclear cells were isolated by Ficoll separation and cryopreserved at $-20^{\circ} \mathrm{C}$ according to NIH Center for Human Immunology (CHI) protocols (https://chi.niaid.nih.gov/web/new/our-research/ SOP-Isolation.pdf). Thawed cells were washed and resuspended in PBS. Viability was assessed using LIVE/ DEAD Aqua fixable viability dye (Life Sciences), followed by a wash in FACS staining buffer (PBS supplemented with $1 \%$ normal mouse serum, $1 \%$ goat serum, $0.02 \%$ sodium azide) (Gemini Bio-Products). Cells were stained according to our previously published protocols (25) for 5 tubes of the CLIP panel (Tregs, Th17, Th1/Th2, B naive/memory, and NK cells) (Supplemental Table 1 and Supplemental Figure 4). Acquisition was performed using a Becton Dickinson LSR Fortessa equipped with 5 lasers (355-nm, 407-nm, 488$\mathrm{nm}, 532-\mathrm{nm}, 633-\mathrm{nm}$ wavelengths) with 22 photomultiplier tube (PMT) detectors. Data were acquired using FACSDiva 6.1.2 software (BD), and we ensured that a minimum of 50,000 CD4 ${ }^{+} \mathrm{T}$ cells was recorded to be able to accurately assess minor cell populations. Further details are as described in Olnes et al. (25).

Luminex assay. Plasma was collected and stored at $-20^{\circ} \mathrm{C}$ per NIH Center for Human Immunology protocols (http://www.nhlbi.nih.gov/resources/chi/documents/SOP-Isolation.pdf). Luminex assays were performed according to previously published methods (25) and per the manufacturer's instructions using kits from Bio-Rad: 27-plex cytokine group I, 21-plex cytokine group II, 10-plex diabetes, 4-plex and 5-plex acute phases. Median fluorescence intensities were collected on a Luminex-100 instrument (Bio-Rad), using Bio-Plex Manager software version 6. Standard curves were generated for each cytokine using lyophilized standards. Cytokine concentrations were determined from standard curves using logistic regression. Samples were run in duplicate and the averages used for analysis. 
Clinical laboratory analyses. Complete blood cell count and differentials, CRP ('CRP.btris,' referring to the hsCRP assay run in the NIH Clinical Center clinical laboratory), and serum lipid levels were quantified using standard clinical laboratory instruments in the NIH Clinical Center (Supplemental Table 1).

Statistics. For study sample size calculation, it was considered that for the change in the percentage of HLA-DR-expressing CD4 ${ }^{+} \mathrm{T}$ cells from baseline to the end of the study (a measurement of interest), a sample size of 20 evaluable participants would provide $82 \%$ power for a 0.05 level 2 -sided $t$ test for the null hypothesis that the mean change is 0 versus the alternative that the mean change is -0.73 , assuming that the standard deviation of the percentage of HLA-DR-expressing $\mathrm{CD}^{+} \mathrm{T}$ cells is 0.778 (20). Outcome measures with either $>10$ missing observations or $<14$ nonzero observations were excluded from analysis; the excluded variables are indicated in Supplemental Table 1. Given the small sample size and skewed distribution for many measures, Wilcoxon's rank-sum test was used to determine measurement differences between normal- and high-hsCRP subjects at baseline. For testing the effect of rosuvastatin treatment and discontinuation, linear mixed models with random intercepts for each subject were used. Treatment effect was assessed by comparing observations after rosuvastatin treatment (days 14, 28, and 42) versus day 0, and discontinuation effect was assessed by comparing observations after treatment discontinuation (day 42) versus observations when treatment was ongoing (days 14 and 28). We first determined whether log transformation was needed for each variable based on Shapiro-Wilk test of normality. The log-transformed data were used in the regression analysis if distribution of the residues was closer to normal after the transformation. Variables that were log transformed are indicated in Supplemental Table 1. All models were run with adjustment for age sex, and race. Luminex assays were run in 2 batches; for each, a standard curve was applied for quantitation. In separate analyses, we also evaluated whether statin effects differed between normal- and highhsCRP subjects (i.e., interactions between rosuvastatin and baseline hsCRP). Spearman's correlation analysis using data from all individuals and all time points was performed using the "rcorr" function of the "Hmisc" package in R. All analyses were performed using R version 3.5.3.

Study approval. After completing informed consent, volunteers were enrolled in \#10-H-0165, an NIH protocol approved and monitored by the NHLBI/NIH Institutional Review Board in accordance with the Declaration of Helsinki and registered under clinicaltrials.gov (NCT01200836).

\section{Author contributions}

PWFK, MS, and MBF analyzed and graphed data and contributed to the writing of the manuscript. SP, $\mathrm{AB}, \mathrm{JC}, \mathrm{FC}, \mathrm{YK}$, and NY designed the study, performed the analyses, analyzed data, and contributed to the writing of the manuscript.

\section{Acknowledgments}

This research was supported by the Intramural Research Program of the NIH, National Institute of Environmental Health Sciences (Z01 ES102005), and the trans-NIH Center for Human Immunology. See supplemental material for CHI Consortium details.

Address correspondence to: Michael B. Fessler, National Institute of Environmental Health Sciences, 111 T.W. Alexander Drive, P.O. Box 12233, MD D2-01, Research Triangle Park, North Carolina 27709, USA. Phone: 984.287.4081; Email: fesslerm@niehs.nih.gov.

SP's present address is: AstraZeneca, Gaithersburg, Maryland, USA.

AB's present address is: Precision Immunology, Immunology \& Inflammation Research Therapeutic Area, Sanofi, Cambridge, Massachusetts, USA.

JC's present address is: Laboratory of Human Carcinogenesis, Center for Cancer Research, National Cancer Institute, NIH, Bethesda, Maryland, USA.

\footnotetext{
1. Salami JA, et al. National trends in statin use and expenditures in the us adult population from 2002 to 2013: insights from the Medical Expenditure Panel Survey. JAMA Cardiol. 2017;2(1):56-65.

2. Parihar SP, Guler R, Brombacher F. Statins: a viable candidate for host-directed therapy against infectious diseases. Nat Rev
} 
Immunol. 2019;19(2):104-117.

3. Gabor KA, Fessler MB. Roles of the meautvalonate pathway and cholesterol trafficking in pulmonary host defense. Curr Mol Pharmacol. 2017;10(1):27-45.

4. Arefieva TI, Filatova AY, Potekhina AV, Shchinova AM. Immunotropic effects and proposed mechanism of action for 3-hydroxy-3-methylglutaryl-coenzyme a reductase inhibitors (statins). Biochemistry Mosc. 2018;83(8):874-889.

5. Blank N, et al. Atorvastatin inhibits T cell activation through 3-hydroxy-3-methylglutaryl coenzyme A reductase without decreasing cholesterol synthesis. J Immunol. 2007;179(6):3613-3621.

6. Bu DX, et al. Statin-induced Krüppel-like factor 2 expression in human and mouse T cells reduces inflammatory and pathogenic responses. J Clin Invest. 2010;120(6):1961-1970.

7. Ghittoni R, et al. Simvastatin inhibits T-cell activation by selectively impairing the function of Ras superfamily GTPases. FASEB J. 2005;19(6):605-607.

8. Ascer E, et al. Atorvastatin reduces proinflammatory markers in hypercholesterolemic patients. Atherosclerosis. 2004;177(1):161-166.

9. Link A, Ayadhi T, Böhm M, Nickenig G. Rapid immunomodulation by rosuvastatin in patients with acute coronary syndrome. Eur Heart J. 2006;27(24):2945-2955.

10. Nixon DE, et al. Effects of atorvastatin on biomarkers of immune activation, inflammation, and lipids in virologically suppressed, human immunodeficiency virus-1-infected individuals with low-density lipoprotein cholesterol \&1t;130 mg/dL (AIDS Clinical Trials Group Study A5275). J Clin Lipidol. 2017;11(1):61-69.

11. Weiss L, et al. Rosuvastatin is effective to decrease CD8 T-cell activation only in HIV-infected patients with high residual T-cell activation under antiretroviral therapy. J Acquir Immune Defic Syndr. 2016;71(4):390-398.

12. Wiklund O, Mattsson-Hultén L, Hurt-Camejo E, Oscarsson J. Effects of simvastatin and atorvastatin on inflammation markers in plasma. J Intern Med. 2002;251(4):338-347.

13. Funderburg NT, et al. Rosuvastatin reduces vascular inflammation and T-cell and monocyte activation in HIV-infected subjects on antiretroviral therapy. J Acquir Immune Defic Syndr. 2015;68(4):396-404

14. Rezaie-Majd A, et al. Simvastatin reduces expression of cytokines interleukin-6, interleukin-8, and monocyte chemoattractant protein-1 in circulating monocytes from hypercholesterolemic patients. Arterioscler Thromb Vasc Biol. 2002;22(7):1194-1199.

15. Albert MA, Danielson E, Rifai N, Ridker PM, PRINCE Investigators. Effect of statin therapy on C-reactive protein levels: the pravastatin inflammation/CRP evaluation (PRINCE): a randomized trial and cohort study. JAMA. 2001;286(1):64-70.

16. Alber HF, et al. Effect of atorvastatin on circulating proinflammatory T-lymphocyte subsets and soluble CD40 ligand in patients with stable coronary artery disease — a randomized, placebo-controlled study. Am Heart J. 2006;151(1):139.

17. Skinner OP, et al. Lack of protein prenylation promotes NLRP3 inflammasome assembly in human monocytes. J Allergy Clin Immunol. 2019;143(6):2315-2317.e3.

18. Akula MK, et al. Control of the innate immune response by the mevalonate pathway. Nat Immunol. 2016;17(8):922-929.

19. Guo C, et al. Cholesterol homeostatic regulator SCAP-SREBP2 integrates NLRP3 inflammasome activation and cholesterol biosynthetic signaling in macrophages. Immunity. 2018;49(5):842-856.e7.

20. Fehr T, et al. Statin-induced immunomodulatory effects on human T cells in vivo. Atherosclerosis. 2004;175(1):83-90.

21. Han KH, et al. HMG-CoA reductase inhibition reduces monocyte CC chemokine receptor 2 expression and monocyte chemoattractant protein-1-mediated monocyte recruitment in vivo. Circulation. 2005;111(11):1439-1447.

22. Rodríguez-Perea AL, Montoya CJ, Olek S, Chougnet CA, Velilla PA. Statins increase the frequency of circulating CD4+ FOXP3+ regulatory T cells in healthy individuals. J Immunol Res. 2015;2015:762506.

23. Ridker PM, et al. Rosuvastatin to prevent vascular events in men and women with elevated C-reactive protein. $N$ Engl J Med. 2008;359(21):2195-2207.

24. Riesen WF, Engler H, Risch M, Korte W, Noseda G. Short-term effects of atorvastatin on C-reactive protein. Eur Heart J. 2002;23(10):794-799.

25. Olnes MJ, et al. Effects of systemically administered hydrocortisone on the human immunome. Sci Rep. 2016;6:23002.

26. Ridker PM. High-sensitivity C-reactive protein: potential adjunct for global risk assessment in the primary prevention of cardiovascular disease. Circulation. 2001;103(13):1813-1818.

27. Shih AW, McFarlane A, Verhovsek M. Haptoglobin testing in hemolysis: measurement and interpretation. Am J Hematol. 2014;89(4):443-447.

28. Weitz-Schmidt G, et al. Statins selectively inhibit leukocyte function antigen-1 by binding to a novel regulatory integrin site. Nat Med. 2001;7(6):687-692.

29. Lin YC, Lin JH, Chou CW, Chang YF, Yeh SH, Chen CC. Statins increase p21 through inhibition of histone deacetylase activity and release of promoter-associated HDAC1/2. Cancer Res. 2008;68(7):2375-2383.

30. Planagumà A, et al. Lovastatin decreases acute mucosal inflammation via 15-epi-lipoxin A4. Mucosal Immunol. 2010;3(3):270-279.

31. Zeiser R. Immune modulatory effects of statins. Immunology. 2018;154(1):69-75.

32. Bruegel M, Teupser D, Haffner I, Mueller M, Thiery J. Statins reduce macrophage inflammatory protein-1alpha expression in human activated monocytes. Clin Exp Pharmacol Physiol. 2006;33(12):1144-1149.

33. Mandosi E, et al. Atorvastatin downregulates monocyte CD36 expression, nuclear NFkappaB and TNFalpha levels in type 2 diabetes. J Atheroscler Thromb. 2010;17(6):539-545.

34. Ferro D, Parrotto S, Basili S, Alessandri C, Violi F. Simvastatin inhibits the monocyte expression of proinflammatory cytokines in patients with hypercholesterolemia. J Am Coll Cardiol. 2000;36(2):427-431.

35. Kiener PA, Davis PM, Murray JL, Youssef S, Rankin BM, Kowala M. Stimulation of inflammatory responses in vitro and in vivo by lipophilic HMG-CoA reductase inhibitors. Int Immunopharmacol. 2001;1(1):105-118.

36. Takahashi HK, Mori S, Iwagaki H, Yoshino T, Tanaka N, Nishibori M. Simvastatin induces interleukin-18 production in human peripheral blood mononuclear cells. Clin Immunol. 2005;116(3):211-216.

37. Kuijk LM, et al. Statin synergizes with LPS to induce IL-1beta release by THP-1 cells through activation of caspase-1. Mol Immunol. 2008;45(8):2158-2165.

38. Liao YH, et al. HMG-CoA reductase inhibitors activate caspase-1 in human monocytes depending on ATP release and P2X7 activation. J Leukoc Biol. 2013;93(2):289-299. 
39. Xu JF, et al. Statins and pulmonary fibrosis: the potential role of NLRP3 inflammasome activation. Am J Respir Crit Care Med. 2012;185(5):547-556.

40. Rogers AJ, et al. Association of elevated plasma interleukin-18 level with increased mortality in a clinical trial of statin treatment for acute respiratory distress syndrome. Crit Care Med. 2019;47(8):1089-1096.

41. Calfee CS, et al. Acute respiratory distress syndrome subphenotypes and differential response to simvastatin: secondary analysis of a randomised controlled trial. Lancet Respir Med. 2018;6(9):691-698.

42. Fessler MB, Rose K, Zhang Y, Jaramillo R, Zeldin DC. Relationship between serum cholesterol and indices of erythrocytes and platelets in the US population. J Lipid Res. 2013;54(11):3177-3188.

43. Olszewska-Banaszczyk M, Jackowska P, Gorzelak-Pabiś P, Pytel E, Koter-Michalak M, Broncel M. Comparison of the effects of rosuvastatin monotherapy and atorvastatin-ezetimibe combined therapy on the structure of erythrocyte membranes in patients with coronary artery disease. Pharmacol Rep. 2018;70(2):258-262.

44. Zhong Y, et al. Total cholesterol content of erythrocyte membranes is associated with the severity of coronary artery disease and the therapeutic effect of rosuvastatin. Ups J Med Sci. 2012;117(4):390-398.

45. Uydu HA, et al. The effects of atorvastatin therapy on rheological characteristics of erythrocyte membrane, serum lipid profile and oxidative status in patients with dyslipidemia. J Membr Biol. 2012;245(11):697-705.

46. Forsyth AM, Braunmüller S, Wan J, Franke T, Stone HA. The effects of membrane cholesterol and simvastatin on red blood cell deformability and ATP release. Microvasc Res. 2012;83(3):347-351.

47. Brookhart MA, Solomon DH, Glynn RJ, Ridker PM. Effect of rosuvastatin on hemoglobin levels in patients with anemia and low-grade inflammation: a post hoc analysis of the JUPITER trial. J Clin Pharmacol. 2011;51(10):1483-1487.

48. Bolaman Z, Kadikoylu G, Ozgel N, Yenisey C. Effects of atorvastatin on coagulation parameters and homocysteine in patients with primary hypercholesterolemia. J Natl Med Assoc. 2006;98(8):1273-1277.

49. Sattar N, et al. Statins and risk of incident diabetes: a collaborative meta-analysis of randomised statin trials. Lancet. 2010;375(9716):735-742.

50. Rajpathak SN, Kumbhani DJ, Crandall J, Barzilai N, Alderman M, Ridker PM. Statin therapy and risk of developing type 2 diabetes: a meta-analysis. Diabetes Care. 2009;32(10):1924-1929.

51. Rowlands J, Heng J, Newsholme P, Carlessi R. Pleiotropic effects of glp-1 and analogs on cell signaling, metabolism, and function. Front Endocrinol (Lausanne). 2018;9:672.

52. DeGorter MK, et al. Clinical and pharmacogenetic predictors of circulating atorvastatin and rosuvastatin concentrations in routine clinical care. Circ Cardiovasc Genet. 2013;6(4):400-408.

53. Verschuren JJ, et al. A systematic review on pharmacogenetics in cardiovascular disease: is it ready for clinical application? Eur Heart J. 2012;33(2):165-175.

54. Hegele RA, Tsimikas S. Lipid-lowering agents. Circ Res. 2019;124(3):386-404.

55. Cao YX, Li S, Liu HH, Li JJ. Impact of PCSK9 monoclonal antibodies on circulating hs-CRP levels: a systematic review and meta-analysis of randomised controlled trials. BMJ Open. 2018;8(9):e022348. 